Verificou-se ainda uma tendência para uma posição mais retrusiva dos lábios superior e inferior em relação às linhas de Ricketts e Burstone no grupo com agenesia de segundos pré-molares. O ângulo ANB e o ângulo IMPA registaram uma redução estatisticamente significativa $(p<0,05)$ no Grupo Agenesia. Foi ainda observada uma ligeira diminuição do ângulo SNA e um ligeiro aumento do ângulo SNB, traduzindo numa ligeira retrusão da maxila e protrusão da mandíbula. Esta informação é reforçada pelo valor médio negativo do Witts, com uma tendência para inversão da relação intermaxilar sagital (classe III). As variáveis dentárias não registaram diferenças estatisticamente significativas. No entanto, houve uma tendência para pro-inclinação do incisivo superior e retro-inclinação do incisivo inferior. Conclusões: Os resultados sugerem que existem algumas alterações significativas características destes indivíduos: o lábio superior é mais curto, a profundidade do sulco labial inferior é menor, os ângulos ANB e IMPA estão significativamente reduzidos e existe uma tendência para uma posição mais retruída dos lábios superior e inferior.

http://doi.org/10.24873/j.rpemd.2022.01.958

\#102 Estabilidade após tratamento ortodôntico-cirúrgico: estudo retrospetivo

João Matos*, Raquel Travassos, Francisco Caramelo, Inês Francisco, Francisco do Vale

Faculdade de Medicina da Universidade de Coimbra, Instituto de Investigação Clínica e Biomédica de Coimbra da FMUC, Instituto de Ortodontia da Faculdade de Medicina da Universidade de Coimbra

Objetivos: Avaliar a estabilidade dentária e esquelética após tratamento ortodôntico-cirúrgico e, secundariamente, analisar o efeito do tempo de contenção, tipo de classe esquelética, sexo e idade do doente no final do tratamento na estabilidade. Materiais e métodos: Para este estudo retrospetivo longitudinal selecionou-se uma amostra de 25 doentes submetidos a tratamento ortodôntico-cirúrgico. A medição das variáveis dentárias e esqueléticas foi realizada com uma régua milimétrica e com o software Dolphin Image, respetivamente. Os modelos de estudo e as telerradiografias de perfil da face foram avaliados em três momentos: antes (T0), imediatamente após a remoção da aparatologia (T1) e na fase de contenção (T2) do tratamento ortodôntico-cirúrgico. Para calcular a diferença das variáveis em análise entre T2 e T1 aplicou-se o teste Wilcoxon, cujo valor p foi corrigido para comparações múltiplas pelo método Benjamini-Hochberg. Para a análise da influência do tempo de contenção usou-se o teste Kruskal Wallis. A associação das variáveis nominais foi realizada pelo teste de Fisher, enquanto que para estimar as diferenças entre variáveis quantitativas optou-se pelo teste Mann-Whitney. Considerou-se como estatisticamente significativos valores para $p<0,05$. Resultados: Não foram registadas diferenças estatisticamente significativas nas variáveis dentárias e esqueléticas $(p>0,05)$ entre os momentos T2 e T1. Não se verificaram diferenças significativas no overjet e no ANB entre os vários intervalos de tempo de contenção (KW, $\mathrm{p}=0.821$ e KW, $\mathrm{p}=0.107$, respetivamente). $\mathrm{O}$ tipo de classe esquelética, sexo e idade no final do tratamento não influenciaram a recidiva do tratamento ortodôntico-cirúrgico (Fisher, $p=0,202$; Fisher, $\mathrm{p}=1,000 ; \mathrm{MW}, \mathrm{p}=0.667$, respetivamente). Registaram-se oito casos de recidiva dentária (32\% - IC95\% [12.4\%; 51.7\%]) e nenhum caso $(0 \%)$ de recidiva esquelética. Conclusões: $\mathrm{O}$ tratamento ortodôntico-cirúrgico foi um tratamento estável a longo prazo para amostra estudada, independentemente do tempo de contenção, tipo de classe esquelética, sexo e idade final do tratamento. Estudos futuros deverão contemplar uma amostra mais robusta e homogénea, que permita a subdivisão representativa dos grupos em análise.

http://doi.org/10.24873/j.rpemd.2022.01.959

\section{\#103 Impacto psicossocial das deformidades dentofaciais em pacientes com necessidade de TOCO}

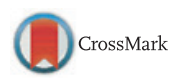

Luana Martins*, Carlos Ferreira de Almeida, Susana Silva

FMDUP, Mestrado Integrado em Medicina Dentária UCP - Viseu

Objetivos: Esta investigação tem como principal objetivo avaliar o impacto na qualidade de vida e autoestima que as deformidades dentofaciais promovem em pacientes com necessidade de Tratamento-Ortodôntico-Cirúrgico-Ortognático (TOCO). Materiais e métodos: Este estudo transversal descritivo avaliou dois grupos de pacientes: grupo de estudo - pacientes com deformidades dentofaciais e com necessidade de TOCO e o grupo controlo - pacientes com necessidade tratamento ortodôntico convencional. A recolha de dados foi feita com dois tipos de questionários, um para avaliar a qualidade de vida (WHOQOL-BREF) e o outro para avaliar a autoestima (Escala de Autoestima de Rosenberg). Resultados: A amostra é constituída por um total de 79 pacientes: o grupo de estudo com 32 pacientes e o grupo controlo com 42 pacientes. No total da amostra a idade varia entre os 17-48 anos e a média das idades é de $25,35( \pm 5,93)$. No que toca ao género $69,62 \%$ são do género feminino e $30,38 \%$ são do género masculino. Relativamente à autoestima a média encontrada foi de 49,94 $( \pm 7,54)$ e por fim a qualidade de vida, onde se obteve uma média no domínio geral de $8,45( \pm 1,07)$, no domínio físico $16,85( \pm 2,14)$, no domínio psicológico 16,53 $( \pm 2,14)$, nas relações sociais de $16,30( \pm 2,78)$ e no meio ambiente de $16,48( \pm 7,54)$. Conclusões: Os pacientes com deformidades dentofaciais mais acentuadas apresentaram uma qualidade de vida e uma autoestima mais baixas em comparação com o grupo controlo. http://doi.org/10.24873/j.rpemd.2022.01.960

\section{\#104 Experiência pedagógica sobre} sustentabilidade com o recurso ferramentas colaborativas

Maria João Ponces*, Marta Jorge, Berta Meireles, Eugénio Martins, Carlos Pires, Saúl Castro

Universidade de Trás-os-Montes e Alto Douro, Faculdade de Medicina Dentária da Universidade do Porto

Objetivos: Este estudo exploratório apresenta os resultados recolhidos a partir de dois questionários instituídos na UC de 
Tecnologias de Informação e Comunicação da Faculdade de Medicina Dentária da Universidade do Porto, cujo intuito principal foi promover competências com ferramenta colaborativas através de um projeto sobre sustentabilidade em Medicina Dentária. Organizados em grupos, os estudantes realizaram trabalhos, que apresentaram em ambiente de sala de aula e construíram um website. O objetivo do estudo foi perceber o nível de utilização das ferramentas e o impacto que o projeto teve na aquisição de competências com as mesmas e de competências comunicacionais e colaborativas em contexto de grupo. Materiais e métodos: Os dados foram recolhidos a partir de 2 questionários elaborados para o efeito e respondidos por 67 estudantes antes do início e no final do projeto. Mediante o teste do Qui-quadrado, assegurou-se haver consistência na amostra que respondeu aos questionários. Estes visavam analisar o conhecimento e a utilização que os estudantes faziam das ferramentas colaborativas da Google e o impacto que o projeto representava na aquisição de competências. Foi efetuada a análise descritiva e uma análise fatorial exploratória que permitiu, entre outras competências transversais, identificar os domínios 'interação estudante-estudante' e 'Interação individual" dividido nos subdomínios: "interação estudante-ferramentas tecnológicas" e "competências comunicacionais". O efeito da participação no projeto foi avaliado comparando as pontuações atribuídas às questões dos $1 .^{\circ}$ e $2 .^{\circ}$ questionários através do Teste de Mann-Whitney. Foi considerado um nível de significância de 5\%. Resultados: Não existiram diferenças estatisticamente significativas entre os estudantes que responderam aos dois questionários. No que concerne à utilização e conhecimento prévio das ferramentas colaborativas, as mais referidas foram o Gmail (100\%) e o Drive (83.6\%), Sites (13.4\%), sendo a menos utilizada a ferramenta Sheets (9\%). Quanto à evolução ao longo do projeto, os resultados mostram uma melhoria estatisticamente significativa nos domínios interação estudante-estudante $(p=0.027)$ e estudante-ferramentas tecnológicas $(p=0.006)$. Conclusões: O projeto revelou-se positivo não somente no que toca à aquisição de competências na utilização das ferramentas comunicacionais, mas também competências colaborativas em contexto grupal.

http://doi.org/10.24873/j.rpemd.2022.01.961

\#105 Aquisição de competências pessoais numa experiência pedagógica sobre sustentabilidade

Maria João Ponces, Marta Jorge, Berta Meireles, Eugénio Martins, Carlos Pires, Saúl Castro*

Universidade de Trás-os-Montes e Alto Douro, Faculdade de Medicina Dentária da Universidade do Porto

Objetivos: Este estudo exploratório apresenta os resultados recolhidos a partir de dois questionários relacionados com uma experiência pedagógica realizada na unidade curricular de Tecnologias de Informação e Comunicação da Faculdade de Medicina Dentária da Universidade do Porto, cujo intuito global foi promover as denominadas soft e hard skill, através de um projeto sobre sustentabilidade em Medicina Dentária. Organizados em grupos, os estudantes realizaram trabalhos so- bre tópicos relacionados com a temática central, que apresentaram em ambiente de sala de aula e, adicionalmente, construíram um website. O objetivo do presente estudo foi perceber o impacto que o projeto apresentou como resultados de aprendizagem, na aquisição de conhecimentos sobre a temática central do projeto. Materiais e métodos: Os dados foram recolhidos a partir de 2 questionários elaborados para o efeito e respondidos por 67 estudantes antes do início e no final do projeto. Mediante o teste do Qui-quadrado, assegurou-se haver consistência na amostra que respondeu a ambos os questionários. Estes visavam analisar a aquisição de competências proporcionadas pelo projeto. Para tal, procedeu-se a uma análise fatorial exploratória com o objetivo de identificar os domínios avaliados pelo questionário no que se refere a competências transversais. O efeito da participação no projeto, nos diferentes domínios foi avaliado comparando as pontuações atribuídas às questões dos $1 .^{\circ}$ e $2 .^{\circ}$ questionários através do Teste de Mann-Whitney. Foi considerado um nível de significância de 5\%. Resultados: Não existiram diferenças estatisticamente significativas entre os estudantes que responderam ao questionário antes e após o projeto. A análise fatorial exploratória levou à identificação entre outros, dos domínios 'interação estudante-instrutor' e 'preocupações ambientais". Quanto à evolução ao longo do projeto, as respostas apresentadas permitiram aferir uma melhoria estatisticamente significativa em ambos os domínios ("interação estudante-instrutor “- $p<0.001$ e "preocupações ambientais"- p = 0.001). Conclusões: O projeto sobre sustentabilidade em Medicina Dentária revelou-se positivo não só no que toca à aquisição de conhecimentos, mas também no que se relaciona com a sensibilização dos estudantes para uma temática tão premente no quotidiano atual.

http://doi.org/10.24873/j.rpemd.2022.01.962

\#106 Determinação da exatidão com duas técnicas
de produção CAD-CAM de coroas provisória

Alexandre Fonseca*, Ricardo Jorge Pinto, Joana Paulos Cabrita, João Carlos Roque, Rodrigo Malheiro, Duarte Marques

Faculdade de Medicina Dentária da Universidade de Lisboa

Objetivos: o objetivo deste estudo in vitro é comparar a exatidão entre coroas provisórias confecionadas sobre um preparo vertical, produzidas pelo método de fresagem e pelo método de impressão-3D. Materiais e métodos: Foi realizado um estudo in vitro com a produção de 10 coroas provisórias por grupo com diferentes métodos CAD-CAM: Grupo 1 - Impressas e Grupo 2- Fresadas. Como referência para os dois grupos foi utilizado um molar mandibular de resina (46) (KaVo EWL model teeth), o qual foi preparado de acordo com métodos previamente descritos, correspondendo a uma preparação sem chanfro de terminação e um desgaste axial de cerca de $0,5 \mathrm{~mm}$ e oclusal de $1 \mathrm{~mm}$ a $1,5 \mathrm{~mm}$. Para obter o desenho da coroa, realizou-se a leitura do molar antes e após o preparo com um digitalizador de laboratório (S600, ZIRKONZAHN), correspondendo a mesma à subtração entre os dois ficheiros de STL. Foram impressas 10 coroas em resina provisória (NextDent ${ }^{\circledR}$ C\% 26B MFH) numa impressora DLP (NextDent ${ }^{\circledR} 5100$ 3D Prin- 